\title{
Non-Spectral Problem of Self-Affine Measures in $\mathbb{R}^{3}$
}

\author{
Yanbo Yuan \\ College of Mathematics and Statistics, Northwest Normal University, Lanzhou, China \\ Email: yuanybmaths@126.com
}

How to cite this paper: Yuan, Y.B. (2021) Non-Spectral Problem of Self-Affine Measures in $\mathbb{R}^{3}$. Advances in Pure Mathematics, 11, 717-734.

https://doi.org/10.4236/apm.2021.118047

Received: July 1, 2021

Accepted: August 9, 2021

Published: August 12, 2021

Copyright $\odot 2021$ by author(s) and Scientific Research Publishing Inc. This work is licensed under the Creative Commons Attribution International License (CC BY 4.0).

http://creativecommons.org/licenses/by/4.0/

\section{(c) (i) Open Access}

\begin{abstract}
The problems of determining the spectrality or non-spectrality of a measure have been received much attention in recent years. One of the non-spectral problems on $\mu_{M, D}$ is to estimate the number of orthogonal exponentials in $L^{2}\left(\mu_{M, D}\right)$. In the present paper, we establish some relations inside the zero set $Z\left(\hat{\mu}_{M, D}\right)$ by the Fourier transform of the self-affine measure $\mu_{M, D}$. Based on these facts, we show that $\mu_{M, D}$ is a non-spectral measure and there exist at most 4 mutually orthogonal exponential functions in $L^{2}\left(\mu_{M, D}\right)$, where the number 4 is the best possible. This extends several known conclusions.
\end{abstract}

\section{Keywords}

Iterated Function System (IFS), Self-Affine Measure, Orthogonal Exponentials

\section{Introduction}

Let $M \in M_{n}(\mathbb{Z})$ be an expanding integer matrix, that is to let one with all eigenvalues $\left|\lambda_{i}(M)\right|>1$ and $D \subset \mathbb{Z}^{n}$ be a finite subset of cardinality $|D|$. The probability measure $\mu_{M, D}$ associated with an iterated function system

$$
\left\{\phi_{d}(x)=M^{-1}(x+d)\right\}_{d \in D}
$$

is uniquely determined. It only depends upon an expanding matrix $M$ and a finite digit set $D$. The attractor $T:=T(M, D)$ is unique non-empty compact set satisfying

$$
M T=\bigcup_{d \in D}(T+d),
$$

and the measure $\mu:=\mu_{R, D}$ is a unique probability measure satisfying the self-affine 
identity

$$
\mu=\frac{1}{|D|} \sum_{d \in D} \mu \circ \phi_{d}^{-1} .
$$

Such $\mu_{M, D}$ is supported on $T(M, D)$ and is called self-affine measure (see [1]).

Recall that for a probability measure $\mu_{M, D}$ of compact support on $\mathbb{R}^{n}$, we call $\mu_{M, D}$ a spectral measure if there exists a discrete set $\Lambda \subset \mathbb{R}^{n}$ such that $E_{\Lambda}:=\left\{e^{2 \pi i\langle\lambda, x\rangle}: \lambda \in \Lambda\right\}$ forms an orthogonal basis for $L^{2}\left(\mu_{M, D}\right)$. The set $\Lambda$ is then called a spectrum for $\mu_{M, D}$.

Spectral measure is a natural generalization of spectral set introduced by Fuglede [2] whose famous conjecture and its related problems have received much attention in recent years (see [3] [4] [5] [6]). The spectral self-affine measure problem at the present day consists in determining conditions under which $\mu_{M, D}$ is a spectral measure, and has been studied in the papers [5] [7] [8] [9] [10] (see also [11] [12] for the main goal). Probably the most interesting question is the spectrality or non-spectrality of self-affine measure $\mu_{M, D}$. We will focus our attention on the following question on the generalized three-dimensional Sierpinski gasket: Under what conditions on $M$ and $D$ is $\mu_{M, D}$ a non-spectral measure? It is known that the non-spectral problem on self-affine measures consists of the following two classes:

1) There are at most a finite number of orthogonal exponentials in $L^{2}\left(\mu_{M, D}\right)$, that is, $\mu_{M, D}$-orthogonal exponentials contains at most finite elements. The main questions here are to estimate the number of orthogonal exponentials in $L^{2}\left(\mu_{M, D}\right)$ and to find them (see [7] [8] [13] [14] [15] [16] [17]).

2) There are natural infinite families of orthogonal exponentials, but none of them forms an orthogonal basis in $L^{2}\left(\mu_{M, D}\right)$. The main question is whether some of these families can be combined to form larger collections of orthogonal exponentials. The other questions concerning this class can be found in [18].

In the present paper, we will consider the questions of the class (I) for the generalized three-dimensional Sierpinski gasket. A fractal F is a set that admits a system of scale transformations; intuitively they have the property that $\mathrm{F}$ looks the same as the scaling is varied. Typically a fractal comes equipped with an invariant measure. However as is illustrated by such familiar cases as the Cantor set and its invariant measure, or one of the Sierpinski examples, one must pass to a limit, and the limit typically allows intricate non-linearities. A popular representation of a class of fractals is realized with a finite set of affine transformations in Euclidean space, and this is the setting for the present paper. Now classical Fourier series relies on linearity, and so asking for Fourier series in the context of fractals is a new framework. The result below indicates the limits one encounters in such an endeavor. The main theorem improves what was previously known, i.e., papers by Dutkay and Jorgensen and by Li.

Recall that the self-affine measure $\mu_{M, D}$ corresponding to 


$$
M=\left[\begin{array}{ccc}
p_{1} & 0 & 0 \\
p_{2} & p_{4} & 0 \\
p_{3} & 0 & p_{5}
\end{array}\right] \text { and } D=\left\{\left(\begin{array}{l}
0 \\
0 \\
0
\end{array}\right),\left(\begin{array}{l}
1 \\
0 \\
0
\end{array}\right),\left(\begin{array}{l}
0 \\
1 \\
0
\end{array}\right),\left(\begin{array}{l}
0 \\
0 \\
1
\end{array}\right)\right\}
$$

is supported on the three-dimensional Sierpinski gasket $T(M, D)$. In the recent paper, Dutkay and Jorgensen [[9]: Theorem 5.1 (iii)] proved that if $p_{2}=p_{3}=0$, $p_{1}=p_{4}=p_{5}=p$ is odd, then $\mu_{M, D}$-orthogonal exponentials contain at most 256 elements. The general case for the non-spectrality of the self-affine measure $\mu_{M, D}$ is not known. This leaves the following open problem.

Question. How about the non-spectrality of $\mu_{M, D}$ if $p_{1}, p_{4}, p_{5} \in 2 \mathbb{Z}+1$ and one or two of the two numbers $p_{2}, p_{3}$ can take any integer value?

Motivated by the previous research, in the present paper we answer the above question mostly. The main result of the paper is the following.

Theorem 1.1. For self-affine measure $\mu_{M, D}$ corresponding to (1.2), if $p_{4}=p_{5}=p$ and $p_{1}, p \in 2 \mathbb{Z}+1 \backslash\{0,-1\}$, then $\mu_{M, D}$ is a non-spectral measure and there exist at most 4 mutually orthogonal exponential functions in $L^{2}\left(\mu_{M, D}\right)$, where the number 4 is the best possible.

In Section 2, we establish some relations inside the zero set of the Fourier transform of the self-affine measure $\mu_{M, D}$. Based on these established facts, we prove Theorem 1.1 in Section 3.

\section{Relations Inside the Zero Set $\mathrm{Z}\left(\hat{\mu}_{M, D}\right)$}

For any $\lambda_{1}, \lambda_{2} \in \Lambda, \quad \lambda_{1} \neq \lambda_{2}$, the orthogonality condition

$$
\left\langle\mathrm{e}^{2 \pi i\left\langle\lambda_{1}, x\right\rangle}, \mathrm{e}^{2 \pi i\left\langle\lambda_{2}, x\right\rangle}\right\rangle_{L^{2}\left(\mu_{M, D}\right)}=\int \mathrm{e}^{\left.2 \pi i \lambda_{1}-\lambda_{2}, x\right\rangle} \mathrm{d} \mu_{M, D}(x)=\hat{\mu}_{M, D}\left(\lambda_{1}-\lambda_{2}\right)=0,
$$

where $\hat{\mu}_{M, D}$ denotes the Fourier transform of $\mu_{M, D}$. From (1.1), we have

$$
\hat{\mu}_{M, D}(\xi)=m_{D}\left(M^{*-1} \xi\right) \hat{\mu}_{M, D}\left(M^{*-1} \xi\right)\left(\xi \in \mathbb{R}^{n}\right),
$$

which yields

$$
\hat{\mu}_{M, D}(\xi)=\prod_{j=1}^{\infty} m_{D}\left(M^{*-j} \xi\right)
$$

by iteration, where

$$
m_{D}(t):=\frac{1}{|D|} \sum_{d \in D} \mathrm{e}^{2 \pi i\langle d, t\rangle}
$$

and $M^{*}$ denotes the conjugate transpose of $M$, in fact $M^{*}=M^{\mathrm{T}}$.

From (2.1), we have

$$
Z\left(\hat{\mu}_{M, D}\right)=\left\{\xi \in \mathbb{R}^{n}: \exists j \in \mathbb{N} \text { such that } m_{D}\left(M^{*-j} \xi\right)=0\right\} .
$$

Furthermore, we have the following.

Proposition 2.1. Let $\Theta_{0}:=\left\{\xi \in \mathbb{R}^{n}: m_{D}(\xi)=0\right\}$. Then

1) $Z\left(\hat{\mu}_{M, D}\right)=\bigcup_{j=1}^{\infty} M^{* j}\left(\Theta_{0}\right)$;

2) $\xi_{0} \in Z\left(\hat{\mu}_{M, D}\right)$ if and only if $-\xi_{0} \in Z\left(\hat{\mu}_{M, D}\right)$. 
In the following, we will restrict our discussion on the special $M$ and $D$ given by (1.2). Then

$$
\Theta_{0}=B_{1} \cup B_{2} \cup B_{3} \text {, }
$$

where

$$
\begin{aligned}
& B_{1}=\left\{\left(\begin{array}{c}
\frac{1}{2}+k_{1} \\
a+k_{2} \\
\frac{1}{2}+a+k_{3}
\end{array}\right): k_{1}, k_{2}, k_{3} \in \mathbb{Z}, a \in \mathbb{R}\right\} \subset \mathbb{R}^{3}, \\
& B_{2}=\left\{\left(\begin{array}{c}
a+k_{2} \\
\frac{1}{2}+k_{1} \\
\frac{1}{2}+a+k_{3}
\end{array}\right): k_{1}, k_{2}, k_{3} \in \mathbb{Z}, a \in \mathbb{R}\right\} \subset \mathbb{R}^{3}
\end{aligned}
$$

and

$$
B_{3}=\left\{\left(\begin{array}{c}
a+k_{2} \\
\frac{1}{2}+a+k_{3} \\
\frac{1}{2}+k_{1}
\end{array}\right): k_{1}, k_{2}, k_{3} \in \mathbb{Z}, a \in \mathbb{R}\right\} \subset \mathbb{R}^{3} .
$$

From Proposition 2.1, the zero set $Z\left(\hat{\mu}_{M, D}\right)$ can be represented as

$$
Z\left(\hat{\mu}_{M, D}\right)=\bigcup_{j=1}^{\infty} M^{* j}\left(\Theta_{0}\right)=\bigcup_{j=1}^{\infty}\left(M^{* j}\left(B_{1}\right) \cup M^{* j}\left(B_{2}\right) \cup M^{* j}\left(B_{3}\right)\right) .
$$

Let $Z_{1}:=M^{* j}\left(B_{1}\right), Z_{2}:=M^{* j}\left(B_{2}\right)$ and $Z_{3}:=M^{* j}\left(B_{3}\right) \quad(j=1,2, \cdots)$. From (2.4) and (2.5), we further have the following.

Proposition 2.2. The sets $Z_{i}, i=1,2,3$ satisfy the following properties:

i) $t \in Z_{i}$ if and only if $-t \in Z_{i}, i=1,2,3$;

ii) $Z\left(\hat{\mu}_{M, D}\right) \cap \mathbb{Z}^{3}=\varnothing$;

iii) if $t=\left(t_{1}, t_{2}, t_{3}\right)^{\mathrm{T}} \in Z_{1} \pm Z_{1}$, then $t_{3}-t_{2} \in \mathbb{Z}$;

iv) if $t=\left(t_{1}, t_{2}, t_{3}\right)^{\mathrm{T}} \in Z_{2} \pm Z_{2}$, then $t_{2} \in \mathbb{Z}$;

v) if $t=\left(t_{1}, t_{2}, t_{3}\right)^{\mathrm{T}} \in Z_{3} \pm Z_{3}$, then $t_{3} \in \mathbb{Z}$.

Proposition 2.3. Let $t=\left(t_{1}, t_{2}, t_{3}\right)^{\mathrm{T}} \in Z_{1} \cup Z_{2} \cup Z_{3}$. Then the following statements hold:
a) if $t \in Z_{1}$, then $t_{3}-t_{2}=\frac{1}{2}+\mathbb{Z}$;
b) if $t \in Z_{2}$, then $t_{2}=\frac{1}{2}+\mathbb{Z}$;
c) if $t \in Z_{3}$, then $t_{3}=\frac{1}{2}+\mathbb{Z}$;
d) if $t_{2} \in \mathbb{Z}$, then $t_{3} \notin \mathbb{Z}$ and $t \in Z_{1} \cup Z_{3}$;
e) if $t_{3} \in \mathbb{Z}$, then $t_{2} \notin \mathbb{Z}$ and $t \in Z_{1} \cup Z_{2}$. 
Proposition 2.4. If $\xi, \eta \in Z_{1}$ and $\xi \pm \eta=\left(\tau_{1}, \tau_{2}, \tau_{3}\right)^{\mathrm{T}} \in Z\left(\hat{\mu}_{M, D}\right)$, then

$$
\xi \pm \eta \in Z_{2} \cup Z_{3}, \tau_{3}-\tau_{2} \in \mathbb{Z} \text {. }
$$

\section{Proof of Theorem 1.1}

If $\lambda_{j}(j=1,2,3,4,5) \in \mathbb{R}^{3}$ are such that the exponential functions

$$
\mathrm{e}^{2 \pi i\left\langle\lambda_{1}, x\right\rangle}, \mathrm{e}^{2 \pi i\left\langle\lambda_{2}, x\right\rangle}, \mathrm{e}^{2 \pi i\left\langle\lambda_{3}, x\right\rangle}, \mathrm{e}^{2 \pi i\left\langle\lambda_{4}, x\right\rangle}, \mathrm{e}^{2 \pi i\left\langle\lambda_{5}, x\right\rangle}
$$

are mutually orthogonal in $L^{2}\left(\mu_{M, D}\right)$, then the differences $\lambda_{j}-\lambda_{i}(1 \leq i \neq j \leq 5)$ are in the zero set $Z\left(\hat{\mu}_{M, D}\right)$. That is, we have

$$
\lambda_{j}-\lambda_{i} \in Z\left(\hat{\mu}_{M, D}\right)=Z_{1} \cup Z_{2} \cup Z_{3}, 1 \leq i \neq j \leq 5 .
$$

Define $\lambda_{j}-\lambda_{k}$ by

$$
\lambda_{j}-\lambda_{i}=\left(x_{j, i}, y_{j, i}, z_{j, i}\right)^{\mathrm{T}} \in \mathbb{R}^{3}, 1 \leq i \neq j \leq 5 .
$$

We shall apply the above three propositions to deduce a contradiction below.

Observe that the following 10 differences:

$$
\begin{gathered}
\lambda_{2}-\lambda_{1}, \lambda_{3}-\lambda_{1}, \lambda_{4}-\lambda_{1}, \lambda_{5}-\lambda_{1}, \\
\lambda_{3}-\lambda_{2}, \lambda_{4}-\lambda_{2}, \lambda_{5}-\lambda_{2}, \\
\lambda_{4}-\lambda_{3}, \lambda_{5}-\lambda_{3}, \\
\lambda_{5}-\lambda_{4},
\end{gathered}
$$

belong to the union of the three sets $Z_{1}, Z_{2}, Z_{3}$. The well-known pigeon hole principle, there is at least one set which contain at least four elements. When $Z_{1}$ contain at least four elements, we can deduce a contradiction in the following. The other cases which $Z_{2}$ or $Z_{3}$ contain at least four elements can be discussed in the similar manner. We divided the proof into the following five typical cases:

Typical case 1. $\lambda_{2}-\lambda_{1}, \lambda_{3}-\lambda_{1}, \lambda_{4}-\lambda_{1}, \lambda_{5}-\lambda_{1} \in Z_{1}$;

Typical case 2. $\lambda_{2}-\lambda_{1}, \lambda_{3}-\lambda_{1}, \lambda_{4}-\lambda_{1} \in Z_{1}$;

Typical case 3. $\lambda_{2}-\lambda_{1}, \lambda_{3}-\lambda_{1} \in Z_{1}$;

Typical case 4. $\lambda_{2}-\lambda_{1} \in Z_{1}$;

Typical case 5 . $\lambda_{j}-\lambda_{1} \notin Z_{1}, j=2,3,4,5$.

We shall give a method to deal with each typical case by considering the above remainder differences. The other cases (by applying Proposition 2.2 and Proposition 2.3) can be discussed in the same manner. Note that each typical case is concluded with a contradiction.

\subsection{Typical Case 1}

In the case, we have

$$
\lambda_{2}-\lambda_{1}, \lambda_{3}-\lambda_{1}, \lambda_{4}-\lambda_{1}, \lambda_{5}-\lambda_{1} \in Z_{1}
$$

Since

$$
\lambda_{j}-\lambda_{i}=\left(\lambda_{j}-\lambda_{1}\right)-\left(\lambda_{i}-\lambda_{1}\right)(2 \leq i<j \leq 5)
$$


hence, by applying Proposition 2.4, this shows that

$$
\lambda_{5}-\lambda_{2}, \lambda_{5}-\lambda_{3}, \lambda_{5}-\lambda_{4}, \lambda_{4}-\lambda_{2}, \lambda_{4}-\lambda_{3}, \lambda_{3}-\lambda_{2} \in Z_{2} \cup Z_{3} .
$$

The well-known pigeon hole principle, there are three differences in (3.4) that belong to the same set $Z_{2}$ or $Z_{3}$. Without loss of generality, we assume that

$$
\lambda_{5}-\lambda_{2}, \lambda_{5}-\lambda_{3}, \lambda_{5}-\lambda_{4} \in Z_{2} \text {. }
$$

The other cases (by applying Proposition 2.2 and Proposition 2.3) can be discussed in the same manner.

Since

$$
\lambda_{3}-\lambda_{2}=\left(\lambda_{5}-\lambda_{2}\right)-\left(\lambda_{5}-\lambda_{3}\right) \in Z_{2}-Z_{2}
$$

and

$$
\lambda_{4}-\lambda_{3}=\left(\lambda_{5}-\lambda_{3}\right)-\left(\lambda_{5}-\lambda_{4}\right) \in Z_{2}-Z_{2},
$$

then, by applying Proposition 2.2(iv) and Proposition 2.3(d), we have

$$
\lambda_{3}-\lambda_{2}, \lambda_{4}-\lambda_{3} \in Z_{3}
$$

and

$$
y_{3,2}, y_{4,3} \in \mathbb{Z}
$$

From

$$
\lambda_{4}-\lambda_{2}=\left(\lambda_{4}-\lambda_{3}\right)-\left(\lambda_{3}-\lambda_{2}\right) \in Z_{3}+Z_{3},
$$

(3.7) and Proposition 2.2(v), we have

$$
y_{4,2}, z_{4,2} \in \mathbb{Z},
$$

which contradicts Proposition 2.3(d) (e). This completes the proof of Typical case 1 .

\subsection{Typical Case 2}

In the case, we have

$$
\lambda_{2}-\lambda_{1}, \lambda_{3}-\lambda_{1}, \lambda_{4}-\lambda_{1} \in Z_{1}
$$

Since

$$
\lambda_{j}-\lambda_{i}=\left(\lambda_{j}-\lambda_{1}\right)-\left(\lambda_{i}-\lambda_{1}\right)(2 \leq i<j \leq 4),
$$

hence, by applying Proposition 2.4, this shows that

$$
\lambda_{4}-\lambda_{2}, \lambda_{4}-\lambda_{3}, \lambda_{3}-\lambda_{2} \in Z_{2} \cup Z_{3}
$$

and

$$
z_{4,2}-y_{4,2} \in \mathbb{Z}, z_{4,3}-y_{4,3} \in \mathbb{Z}, z_{3,2}-y_{3,2} \in \mathbb{Z}
$$

From (3.10), we know that at least one of the two sets $Z_{2}$ and $Z_{3}$, say $Z_{3}$, contains two differences, say $\lambda_{4}-\lambda_{2}$ and $\lambda_{4}-\lambda_{3}$. The other cases can be discussed in the same manner.

Since

$$
\lambda_{3}-\lambda_{2}=\left(\lambda_{4}-\lambda_{2}\right)-\left(\lambda_{4}-\lambda_{3}\right) \in Z_{3}-Z_{3}
$$


then, by applying Proposition 2.2(v), we have

$$
z_{3,2} \in \mathbb{Z} \text {. }
$$

From (3.11), (3.12) and Proposition 2.3(d) (e), we immediately deduce a contradiction. This completes the proof of Typical case 2 .

\subsection{Typical Case 3}

In the case, we have $\lambda_{2}-\lambda_{1}, \lambda_{3}-\lambda_{1} \in Z_{1}$. Since $\lambda_{3}-\lambda_{2}=\left(\lambda_{3}-\lambda_{1}\right)-\left(\lambda_{2}-\lambda_{1}\right)$, then, by applying Proposition 2.4, this shows that

$$
\lambda_{3}-\lambda_{2} \in Z_{2} \cup Z_{3}
$$

and

$$
z_{3,2}-y_{3,2} \in \mathbb{Z}
$$

We only discuss the case $\lambda_{3}-\lambda_{2} \in Z_{2}$, the other case that $\lambda_{3}-\lambda_{2} \in Z_{3}$ can be discussed in the similar manner.

Now, consider the following differences

$$
\lambda_{5}-\lambda_{2}, \lambda_{5}-\lambda_{3}, \lambda_{5}-\lambda_{4}, \lambda_{4}-\lambda_{2}, \lambda_{4}-\lambda_{3},
$$

then there are at least two differences in (3.14) that belong to the set $Z_{1}$. In fact, any two differences of (3.14) cannot belong to the same set $Z_{1}$. We only discuss the following two cases.

Case 3.1. $\lambda_{4}-\lambda_{2}, \lambda_{5}-\lambda_{4} \in Z_{1}$;

Case 3.2. $\lambda_{5}-\lambda_{2}, \lambda_{5}-\lambda_{3} \in Z_{1}$.

The other cases can be discussed in the similar manner.

1) In Case 3.1, Since

$$
\begin{aligned}
& \lambda_{4}-\lambda_{1}=\left(\lambda_{2}-\lambda_{1}\right)+\left(\lambda_{4}-\lambda_{2}\right) \in Z_{1}+Z_{1}, \\
& \lambda_{5}-\lambda_{4}=\left(\lambda_{5}-\lambda_{2}\right)-\left(\lambda_{4}-\lambda_{2}\right) \in Z_{1}-Z_{1}
\end{aligned}
$$

and

$$
\lambda_{5}-\lambda_{1}=\left(\lambda_{5}-\lambda_{2}\right)+\left(\lambda_{2}-\lambda_{1}\right) \in Z_{1}+Z_{1}
$$

then, by applying Proposition 2.4, we have

$$
\lambda_{4}-\lambda_{1}, \lambda_{5}-\lambda_{4}, \lambda_{5}-\lambda_{1} \in Z_{2} \cup Z_{3}
$$

and

$$
z_{4,1}-y_{4,1}, z_{5,4}-y_{5,4}, z_{5,1}-y_{5,1} \in \mathbb{Z}
$$

Further, at least two differences of (3.14) belong to the same set $Z_{2}$ or $Z_{3}$. Without loss of generality, we assume that

$$
\lambda_{4}-\lambda_{1}, \lambda_{5}-\lambda_{4} \in Z_{3} \text {. }
$$

The other cases can be discussed in the following same manner.

Since $\lambda_{5}-\lambda_{1}=\left(\lambda_{5}-\lambda_{4}\right)+\left(\lambda_{4}-\lambda_{1}\right) \in Z_{3}+Z_{3}$, hence, from (3.16) and Proposition 2.2(v), we have $y_{5,1}, z_{5,1} \in \mathbb{Z}$, which contradicts Proposition 2.3(d) (e). This completes the proof of Case 3.1.

2) In Case 3.2, Since $\lambda_{5}-\lambda_{1}=\left(\lambda_{5}-\lambda_{2}\right)+\left(\lambda_{2}-\lambda_{1}\right) \in Z_{1}+Z_{1}$, therefore, by ap- 
plying Proposition 2.4, we have

$$
\lambda_{5}-\lambda_{1} \in Z_{2} \cup Z_{3} \text { and } z_{5,1}-y_{5,1} \in \mathbb{Z} \text {. }
$$

We divide the discussion of case 3.2 into the following two cases according to (3.18).

Case 3.2.1. $\lambda_{5}-\lambda_{1} \in Z_{2}$;

Case 3.2.2. $\lambda_{5}-\lambda_{1} \in Z_{3}$.

i) In Case 3.2.1, we consider the above remainder differences $\lambda_{4}-\lambda_{1}, \lambda_{5}-\lambda_{4}, \lambda_{4}-\lambda_{2}, \lambda_{4}-\lambda_{3}$. Firstly,

$$
\lambda_{5}-\lambda_{4} \text { cannot belong to the set (or small box) } Z_{1} \text {. }
$$

The reason is as follows.

If $\lambda_{5}-\lambda_{4} \in Z_{1}$, then, it follows that

$$
\begin{aligned}
& \lambda_{4}-\lambda_{2}=\left(\lambda_{5}-\lambda_{2}\right)-\left(\lambda_{5}-\lambda_{4}\right) \in Z_{1}-Z_{1}, \\
& \lambda_{4}-\lambda_{3}=\left(\lambda_{5}-\lambda_{3}\right)-\left(\lambda_{5}-\lambda_{4}\right) \in Z_{1}-Z_{1}
\end{aligned}
$$

and Proposition 2.4, we have

$$
\lambda_{4}-\lambda_{2}, \lambda_{4}-\lambda_{3} \in Z_{2} \cup Z_{3}
$$

and

$$
z_{4,2}-y_{4,2}, z_{4,3}-y_{4,3} \in \mathbb{Z} \text {. }
$$

This is impossible. Otherwise, If $\lambda_{4}-\lambda_{2} \in Z_{2}$, then, from $\lambda_{4}-\lambda_{3}=\left(\lambda_{4}-\lambda_{2}\right)-\left(\lambda_{3}-\lambda_{2}\right) \in Z_{2}-Z_{2}$, (3.21) and Proposition 2.2(iv), we have $y_{4,3}, z_{4,3} \in \mathbb{Z}$, which contradicts Proposition 2.3(d) (e).

Similarly, we have $\lambda_{4}-\lambda_{3} \notin Z_{2}, \lambda_{4}-\lambda_{2}, \lambda_{4}-\lambda_{3} \notin Z_{3}$.

Secondly, for the similar discussion, we have the following facts that:

$\lambda_{4}-\lambda_{3}$ cannot belong to the set (or small box) $Z_{1}$;

$\lambda_{4}-\lambda_{2}$ cannot belong to the set (or small box) $Z_{1}$.

Hence

$$
\lambda_{4}-\lambda_{1}, \lambda_{5}-\lambda_{4}, \lambda_{4}-\lambda_{2}, \lambda_{4}-\lambda_{3} \in Z_{2} \cup Z_{3} \text {. }
$$

The well-known pigeon hole principle, there are at least two differences in (3.24) that belong to the same set $Z_{2}$ or $Z_{3}$. This is impossible.

Claim 3.1 Any three differences of (3.24) can not belong to the same set $Z_{3}$.

Claim 3.1 can be checked directly. For example, if $\lambda_{4}-\lambda_{1}, \lambda_{5}-\lambda_{4}, \lambda_{4}-\lambda_{2} \in Z_{3}$, then, from

$$
\begin{gathered}
\lambda_{2}-\lambda_{1}=\left(\lambda_{4}-\lambda_{1}\right)-\left(\lambda_{4}-\lambda_{2}\right) \in Z_{3}-Z_{3}, \\
\lambda_{5}-\lambda_{2}=\left(\lambda_{5}-\lambda_{4}\right)+\left(\lambda_{4}-\lambda_{2}\right) \in Z_{3}+Z_{3}, \\
\lambda_{5}-\lambda_{1}=\left(\lambda_{5}-\lambda_{4}\right)+\left(\lambda_{4}-\lambda_{1}\right) \in Z_{3}+Z_{3} \text { and Proposition } 2.2(v), \text { we have } \\
z_{2,1}, z_{5,2}, z_{5,1} \in \mathbb{Z} .
\end{gathered}
$$

Since $\lambda_{5}-\lambda_{1}=\left(\lambda_{5}-\lambda_{2}\right)+\left(\lambda_{2}-\lambda_{1}\right) \in Z_{1}+Z_{1}$, hence, by applying Proposition 2.4 , this shows that

$$
z_{5,1}-y_{5,1} \in \mathbb{Z}
$$


From (3.25) and (3.26), we have $y_{5,1}, z_{5,1} \in \mathbb{Z}$, which contradicts Proposition $2.3(\mathrm{~d})(\mathrm{e})$.

Claim 3.2 Any two differences of (3.24) can not belong to the same set $Z_{2}$. Claim 3.2 can be checked directly. For example, if $\lambda_{4}-\lambda_{3}, \lambda_{4}-\lambda_{2} \in Z_{2}$, then, from

$$
\begin{gathered}
\lambda_{3}-\lambda_{2}=\left(\lambda_{4}-\lambda_{2}\right)-\left(\lambda_{4}-\lambda_{3}\right) \in Z_{2}-Z_{2}, \\
\lambda_{4}-\lambda_{2}=\left(\lambda_{4}-\lambda_{3}\right)+\left(\lambda_{3}-\lambda_{2}\right) \in Z_{2}+Z_{2}, \\
\lambda_{4}-\lambda_{3}=\left(\lambda_{4}-\lambda_{2}\right)-\left(\lambda_{3}-\lambda_{2}\right) \in Z_{2}-Z_{2} \text { and Proposition 2.2(iv), we have } \\
y_{3,2}, y_{4,2}, y_{4,3} \in \mathbb{Z} .
\end{gathered}
$$

Since $\lambda_{3}-\lambda_{2}=\left(\lambda_{4}-\lambda_{2}\right)-\left(\lambda_{4}-\lambda_{3}\right) \in Z_{3}+Z_{3}$, hence, by applying Proposition $2.2(\mathrm{v})$, this shows that

$$
z_{3,2} \in \mathbb{Z}
$$

From (3.27) and (3.28), we have $y_{3,2}, z_{3,2} \in \mathbb{Z}$, which contradicts Proposition $2.3(\mathrm{~d})(\mathrm{e})$.

According to the above discussion, $\lambda_{5}-\lambda_{1} \notin Z_{2}$, this completes the proof of Case 3.2.1.

ii) In Case 3.2.2, we consider the above remainder differences $\lambda_{4}-\lambda_{1}, \lambda_{5}-\lambda_{4}, \lambda_{4}-\lambda_{2}, \lambda_{4}-\lambda_{3}$. Firstly,

$$
\lambda_{5}-\lambda_{4} \text { cannot belong to the set (or small box) } Z_{1} \text {. }
$$

The reason is as follows.

If $\lambda_{5}-\lambda_{4} \in Z_{1}$, then, it follows that

$$
\begin{aligned}
& \lambda_{4}-\lambda_{2}=\left(\lambda_{5}-\lambda_{2}\right)-\left(\lambda_{5}-\lambda_{4}\right) \in Z_{1}-Z_{1}, \\
& \lambda_{4}-\lambda_{3}=\left(\lambda_{5}-\lambda_{3}\right)-\left(\lambda_{5}-\lambda_{4}\right) \in Z_{1}-Z_{1}
\end{aligned}
$$

and Proposition 2.4, we have

$$
\lambda_{4}-\lambda_{2}, \lambda_{4}-\lambda_{3} \in Z_{2} \cup Z_{3}
$$

and

$$
z_{4,2}-y_{4,2}, z_{4,3}-y_{4,3} \in \mathbb{Z}
$$

This is impossible. Otherwise, If $\lambda_{4}-\lambda_{2} \in Z_{2}$, then, from $\lambda_{4}-\lambda_{3}=\left(\lambda_{4}-\lambda_{2}\right)-\left(\lambda_{3}-\lambda_{2}\right) \in Z_{2}-Z_{2}$, (3.31) and Proposition 2.2(iv), we have $y_{4,3}, z_{4,3} \in \mathbb{Z}$, which contradicts Proposition 2.3(d) (e).

If $\lambda_{4}-\lambda_{3} \in Z_{2}$, then, from $\lambda_{4}-\lambda_{2}=\left(\lambda_{4}-\lambda_{3}\right)+\left(\lambda_{3}-\lambda_{2}\right) \in Z_{2}+Z_{2}$, (3.31) and Proposition $2.2\left(\right.$ iv), we have $y_{4,2}, z_{4,2} \in \mathbb{Z}$, which contradicts Proposition $2.3(\mathrm{~d})(\mathrm{e})$.

If $\lambda_{4}-\lambda_{2}, \lambda_{4}-\lambda_{3} \in Z_{3}$, then, from $\lambda_{3}-\lambda_{2}=\left(\lambda_{4}-\lambda_{2}\right)-\left(\lambda_{4}-\lambda_{3}\right) \in Z_{3}-Z_{3}$, (3.31) and Proposition 2.2(v), we have $y_{3,2}, z_{3,2} \in \mathbb{Z}$, which contradicts Proposition 2.3(d) (e).

Secondly, for the similar discussion, we have the following facts that:

$$
\lambda_{4}-\lambda_{3} \text { cannot belong to the set (or small box) } Z_{1} \text {; }
$$


$\lambda_{4}-\lambda_{2}$ cannot belong to the set (or small box) $Z_{1}$.

Hence

$$
\lambda_{4}-\lambda_{1}, \lambda_{5}-\lambda_{4}, \lambda_{4}-\lambda_{2}, \lambda_{4}-\lambda_{3} \in Z_{2} \cup Z_{3} .
$$

The well-known pigeon hole principle, there are at least two differences in (3.34) that belong to the same set $Z_{2}$ or $Z_{3}$.

Claim 3.3 Any three differences of (3.34) can not belong to the same set $Z_{3}$. Claim 3.3 can be checked directly. For example, if $\lambda_{4}-\lambda_{1}, \lambda_{5}-\lambda_{4}, \lambda_{4}-\lambda_{2} \in Z_{3}$, then, from

$$
\begin{aligned}
& \lambda_{5}-\lambda_{2}=\left(\lambda_{5}-\lambda_{4}\right)+\left(\lambda_{4}-\lambda_{2}\right) \in Z_{3}+Z_{3}, \\
& \lambda_{2}-\lambda_{1}=\left(\lambda_{4}-\lambda_{1}\right)-\left(\lambda_{4}-\lambda_{2}\right) \in Z_{3}-Z_{3}
\end{aligned}
$$

and Proposition 2.2(v), we have

$$
z_{2,1}, z_{5,2} \in \mathbb{Z}
$$

Since $\lambda_{5}-\lambda_{1}=\left(\lambda_{5}-\lambda_{2}\right)+\left(\lambda_{2}-\lambda_{1}\right) \in Z_{1}+Z_{1}$, hence, by applying Proposition 2.4 , this shows that

$$
z_{5,1}-y_{5,1} \in \mathbb{Z}
$$

From (3.35) and (3.36), we have $y_{5,1}, z_{5,1} \in \mathbb{Z}$, which contradicts Proposition $2.3(\mathrm{~d})(\mathrm{e})$.

Claim 3.4 Any two differences of (3.34) can not belong to the same set $Z_{2}$.

Claim 3.4 can be checked directly. For example, if $\lambda_{5}-\lambda_{4}, \lambda_{4}-\lambda_{2} \in Z_{2}$, then, from

$$
\begin{aligned}
& \lambda_{5}-\lambda_{2}=\left(\lambda_{5}-\lambda_{4}\right)+\left(\lambda_{4}-\lambda_{2}\right) \in Z_{2}+Z_{2}, \\
& \lambda_{4}-\lambda_{3}=\left(\lambda_{4}-\lambda_{2}\right)-\left(\lambda_{3}-\lambda_{2}\right) \in Z_{2}-Z_{2}
\end{aligned}
$$

and Proposition 2.2(iv), we have

$$
y_{5,2}, y_{4,3} \in \mathbb{Z} \text {. }
$$

Obviously, $\lambda_{4}-\lambda_{3} \in Z_{3}$. Let

$$
\left(\lambda_{5}-\lambda_{1}\right)-\left(\lambda_{4}-\lambda_{3}\right)=\left(\xi_{1}, \xi_{2}, \xi_{3}\right)^{\mathrm{T}},\left(\lambda_{3}-\lambda_{1}\right)+\left(\lambda_{5}-\lambda_{4}\right)=\left(\eta_{1}, \eta_{2}, \eta_{3}\right)^{\mathrm{T}} .
$$

By applying Proposition 2.2(v) and Proposition 2.3(e), we have

$$
\xi_{2} \notin \mathbb{Z} \text {. }
$$

By applying Proposition 2.2, Proposition 2.3 and Proposition 2.4, we have

$$
\eta_{2} \in \mathbb{Z} \text {. }
$$

Namely $\left(\lambda_{5}-\lambda_{1}\right)-\left(\lambda_{4}-\lambda_{3}\right) \neq\left(\lambda_{3}-\lambda_{1}\right)+\left(\lambda_{5}-\lambda_{4}\right)$, we immediately deduce a contradiction. This completes the proof of Case 3.2.2.

According to the above discussion, this completes the proof of Typical case 3.

\subsection{Typical Case 4}

In Typical case 4 , we have $\lambda_{2}-\lambda_{1} \in Z_{1}$. We see that in this case, each set contains elements (or differences) in the following Box 2: 


\begin{tabular}{ccc}
\hline$Z_{1}$ & $Z_{2}$ & $Z_{3}$ \\
\hline$\lambda_{2}-\lambda_{1}$ & & \\
\hline
\end{tabular}

Box 1

Since $\lambda_{5}-\lambda_{1}, \lambda_{4}-\lambda_{1}, \lambda_{3}-\lambda_{1} \in Z_{2} \cup Z_{3}$, then, there are at least two differences that belong to the same set $Z_{2}$ or $Z_{3}$. We only discuss the following two cases:

Case 4.1. $\lambda_{5}-\lambda_{1}, \lambda_{3}-\lambda_{1} \in Z_{3}$;

Case 4.2. $\lambda_{5}-\lambda_{1}, \lambda_{4}-\lambda_{1}, \lambda_{3}-\lambda_{1} \in Z_{2}$.

The other cases can be discussed in the similar manner.

1) In Case 4.1 , since $\lambda_{5}-\lambda_{3}=\left(\lambda_{5}-\lambda_{1}\right)-\left(\lambda_{3}-\lambda_{1}\right) \in Z_{3}-Z_{3}$, so, by applying Proposition 2.2(v) and Proposition 2.3(e), we have $\lambda_{5}-\lambda_{3} \in Z_{1} \cup Z_{2}$. There are the following two cases.

Case 4.1.1. $\lambda_{5}-\lambda_{3} \in Z_{1}$;

Case 4.1.2. $\lambda_{5}-\lambda_{3} \in Z_{2}$.

i) In Case 4.1.1, we consider the above remainder differences

$$
\lambda_{5}-\lambda_{4}, \lambda_{5}-\lambda_{2}, \lambda_{4}-\lambda_{2}, \lambda_{4}-\lambda_{3}, \lambda_{3}-\lambda_{2},
$$

then, there are at least two differences that belong to the same set $Z_{1}$. This is impossible.

Claim 4.1 Any two differences of the above remainder differences can not belong to the same set $Z_{1}$.

We only check Claim 4.1 in the following two cases:

Case 4.1.1.1. $\lambda_{5}-\lambda_{4}, \lambda_{3}-\lambda_{2} \in Z_{1}$;

Case 4.1.1.2. $\lambda_{4}-\lambda_{2}, \lambda_{3}-\lambda_{2} \in Z_{1}$.

The other cases can be discussed in the similar manner.

i') In Case 4.1.1.1, since

$$
\begin{aligned}
& \lambda_{4}-\lambda_{3}=\left(\lambda_{5}-\lambda_{3}\right)-\left(\lambda_{5}-\lambda_{4}\right) \in Z_{1}-Z_{1}, \\
& \lambda_{3}-\lambda_{1}=\left(\lambda_{3}-\lambda_{2}\right)+\left(\lambda_{2}-\lambda_{1}\right) \in Z_{1}+Z_{1}
\end{aligned}
$$

and $\lambda_{5}-\lambda_{2}=\left(\lambda_{5}-\lambda_{3}\right)+\left(\lambda_{3}-\lambda_{2}\right) \in Z_{1}+Z_{1}$, therefore, from Proposition 2.4, we have

$$
\lambda_{4}-\lambda_{3} \in Z_{2} \cup Z_{3}
$$

and

$$
z_{4,3}-y_{4,3}, z_{3,1}-y_{3,1} \in \mathbb{Z} \text {. }
$$

If $\lambda_{4}-\lambda_{3} \in Z_{2}$, from $\lambda_{3}-\lambda_{1}=\left(\lambda_{4}-\lambda_{1}\right)-\left(\lambda_{4}-\lambda_{3}\right) \in Z_{2}-Z_{2}$, (3.39) and Proposition 2.2(iv), we have $Z_{3,1}, y_{3,1} \in \mathbb{Z}$, which contradicts Proposition 2.3(d) (e). If $\lambda_{4}-\lambda_{3} \in Z_{3}$, from $\lambda_{4}-\lambda_{1}=\left(\lambda_{4}-\lambda_{3}\right)+\left(\lambda_{3}-\lambda_{1}\right) \in Z_{3}+Z_{3}$, (3.39) and Proposition 2.2(v), we have $z_{4,1}, y_{4,1} \in \mathbb{Z}$, which contradicts Proposition 2.3(d) (e). Therefore, $\lambda_{5}-\lambda_{4}, \lambda_{3}-\lambda_{2} \notin Z_{1}$.

ii') In Case 4.1.1.2, since

$$
\begin{aligned}
& \lambda_{4}-\lambda_{3}=\left(\lambda_{4}-\lambda_{2}\right)-\left(\lambda_{3}-\lambda_{2}\right) \in Z_{1}-Z_{1}, \\
& \lambda_{5}-\lambda_{2}=\left(\lambda_{5}-\lambda_{3}\right)+\left(\lambda_{3}-\lambda_{2}\right) \in Z_{1}+Z_{1},
\end{aligned}
$$




$$
\begin{aligned}
& \lambda_{3}-\lambda_{1}=\left(\lambda_{3}-\lambda_{2}\right)+\left(\lambda_{2}-\lambda_{1}\right) \in Z_{1}+Z_{1} \\
& \text { and } \lambda_{4}-\lambda_{1}=\left(\lambda_{4}-\lambda_{2}\right)+\left(\lambda_{2}-\lambda_{1}\right) \in Z_{1}+Z_{1},
\end{aligned}
$$

hence, from Proposition 2.4, we have

$$
\lambda_{4}-\lambda_{3} \in Z_{2} \cup Z_{3} \text { and } z_{4,3}-y_{4,3}, z_{3,1}-y_{3,1}, z_{4,1}-y_{4,1} \in \mathbb{Z} \text {. }
$$

If $\lambda_{4}-\lambda_{3} \in Z_{2}$, from $\lambda_{3}-\lambda_{1}=\left(\lambda_{4}-\lambda_{1}\right)-\left(\lambda_{4}-\lambda_{3}\right) \in Z_{2}-Z_{2}$, (3.40) and Proposition 2.2(iv), we have $z_{3,1}, y_{3,1} \in \mathbb{Z}$, which contradicts Proposition 2.3(d) (e).

If $\lambda_{4}-\lambda_{3} \in Z_{3}$, from $\lambda_{4}-\lambda_{3}=\left(\lambda_{4}-\lambda_{1}\right)-\left(\lambda_{3}-\lambda_{1}\right) \in Z_{3}-Z_{3}$, (3.40) and Proposition 2.2(v), we have $z_{4,3}, y_{4,3} \in \mathbb{Z}$, which contradicts Proposition 2.3(d) (e). Therefore, $\lambda_{4}-\lambda_{2}, \lambda_{3}-\lambda_{2} \notin Z_{1}$.

ii) In Case 4.1.2, we consider the above remainder differences

$$
\lambda_{5}-\lambda_{4}, \lambda_{5}-\lambda_{2}, \lambda_{4}-\lambda_{2}, \lambda_{4}-\lambda_{3}, \lambda_{3}-\lambda_{2},
$$

then, there are at least three differences that belong to the same set $Z_{1}$. This is impossible.

Claim 4.2 Any three differences of the above remainder differences can not belong to the same set $Z_{1}$.

We only check Claim 4.2 in the following.

Case 4.1.2.1. $\lambda_{5}-\lambda_{4}, \lambda_{3}-\lambda_{2}, \lambda_{4}-\lambda_{2} \in Z_{1}$.

The other cases can be discussed in the similar manner.

iii') In Case 4.1.2.1, since

$$
\begin{aligned}
& \lambda_{4}-\lambda_{3}=\left(\lambda_{4}-\lambda_{2}\right)-\left(\lambda_{3}-\lambda_{2}\right) \in Z_{1}-Z_{1}, \\
& \lambda_{3}-\lambda_{1}=\left(\lambda_{3}-\lambda_{2}\right)+\left(\lambda_{2}-\lambda_{1}\right) \in Z_{1}+Z_{1}
\end{aligned}
$$

and $\lambda_{4}-\lambda_{1}=\left(\lambda_{4}-\lambda_{2}\right)+\left(\lambda_{2}-\lambda_{1}\right) \in Z_{1}+Z_{1}$, therefore, from Proposition 2.4, we have

$$
\lambda_{4}-\lambda_{3} \in Z_{2} \cup Z_{3} \text { and } z_{4,3}-y_{4,3}, z_{3,1}-y_{3,1}, z_{4,1}-y_{4,1} \in \mathbb{Z} .
$$

This is impossible. Otherwise, if $\lambda_{4}-\lambda_{3} \in Z_{2}$ or $Z_{3}$, then, from Proposition 2.2 and Proposition 2.3, we can deduce a contradiction.

Therefore, $\lambda_{5}-\lambda_{3} \notin Z_{2}$. This completes the proof of Case 4.1.

2) In Case 4.2 , since

$$
\begin{aligned}
& \lambda_{5}-\lambda_{3}=\left(\lambda_{5}-\lambda_{1}\right)-\left(\lambda_{3}-\lambda_{1}\right) \in Z_{2}-Z_{2}, \\
& \lambda_{5}-\lambda_{4}=\left(\lambda_{5}-\lambda_{1}\right)-\left(\lambda_{4}-\lambda_{1}\right) \in Z_{2}-Z_{2}
\end{aligned}
$$

and $\lambda_{4}-\lambda_{3}=\left(\lambda_{4}-\lambda_{1}\right)-\left(\lambda_{3}-\lambda_{1}\right) \in Z_{2}-Z_{2}$, then, from Proposition 2.2(iv), we have

$$
\lambda_{5}-\lambda_{4}, \lambda_{4}-\lambda_{3}, \lambda_{5}-\lambda_{3} \in Z_{1} \cup Z_{3} \text { and } y_{5,3}, y_{5,4}, y_{4,3} \in \mathbb{Z} .
$$

Then there are at least two differences that belong to the same set $Z_{1}$ or $Z_{3}$.

In fact, any two differences in (3.41) can not belong to the same set $Z_{1}$ or $Z_{3}$. The reason is as follows.

Claim 4.3 Any two differences of the above remainder differences can not belong to the same set $Z_{1}$.

Claim 4.3 can be checked directly. For example, if $\lambda_{5}-\lambda_{3}, \lambda_{4}-\lambda_{3} \in Z_{1}$, then, 
from $\lambda_{5}-\lambda_{4}=\left(\lambda_{5}-\lambda_{3}\right)-\left(\lambda_{4}-\lambda_{3}\right) \in Z_{1}-Z_{1}$ Proposition 2.4 and (3.41), we have $y_{5,4}, z_{5,4} \in \mathbb{Z}$,

which contradicts Proposition 2.3(d) (e).

Claim 4.4 Any two differences of the above remainder differences can not belong to the same set $Z_{3}$.

Claim 4.4 can be checked directly. For example, if $\lambda_{5}-\lambda_{3}, \lambda_{4}-\lambda_{3} \in Z_{3}$, then, from $\lambda_{5}-\lambda_{4}=\left(\lambda_{5}-\lambda_{3}\right)-\left(\lambda_{4}-\lambda_{3}\right) \in Z_{3}-Z_{3}$, Proposition 2.2(v) and (3.41), we have $y_{5,4}, z_{5,4} \in \mathbb{Z}$, which contradicts Proposition 2.3(d) (e).

According to the above discussion, this completes the proof of Typical case 4.

\subsection{Typical Case 5}

In the case, we have

$$
\lambda_{j}-\lambda_{1} \notin Z_{1}(j=2,3,4,5)
$$

Namely

$$
\lambda_{5}-\lambda_{1}, \lambda_{4}-\lambda_{1}, \lambda_{3}-\lambda_{1}, \lambda_{2}-\lambda_{1} \in Z_{2} \cup Z_{3} .
$$

then, from the well-known pigeon hole principle, there are at least two differences which belong to the same set $Z_{2}$ or $Z_{3}$. This is impossible.

Claim 5.1 Any three differences of the above four differences can not belong to the same set $Z_{2}$ or $Z_{3}$.

Claim 5.1 can be checked directly. For example, if $\lambda_{5}-\lambda_{1}, \lambda_{4}-\lambda_{1}, \lambda_{3}-\lambda_{1} \in Z_{2}$, then, from

$$
\begin{gathered}
\lambda_{5}-\lambda_{4}=\left(\lambda_{5}-\lambda_{1}\right)-\left(\lambda_{4}-\lambda_{1}\right) \in Z_{2}-Z_{2}, \\
\lambda_{5}-\lambda_{3}=\left(\lambda_{5}-\lambda_{1}\right)-\left(\lambda_{3}-\lambda_{1}\right) \in Z_{2}-Z_{2} \\
\lambda_{4}-\lambda_{3}=\left(\lambda_{4}-\lambda_{1}\right)-\left(\lambda_{3}-\lambda_{1}\right) \in Z_{2}-Z_{2} \text { and Proposition 2.2(iv), we have } \\
\lambda_{5}-\lambda_{4}, \lambda_{4}-\lambda_{3}, \lambda_{5}-\lambda_{3} \in Z_{1} \cup Z_{3}
\end{gathered}
$$

and

$$
y_{5,3}, y_{5,4}, y_{4,3} \in \mathbb{Z} \text {. }
$$

Then there are at least two differences which belong to the same set $Z_{1}$ or $Z_{3}$. We only discuss the following two cases:

Case 5.1. $\lambda_{5}-\lambda_{3}, \lambda_{4}-\lambda_{3} \in Z_{1}$;

Case 5.2. $\lambda_{5}-\lambda_{3}, \lambda_{4}-\lambda_{3} \in Z_{3}$.

The other cases can be discussed in the similar manner.

1) If $\lambda_{5}-\lambda_{3}, \lambda_{4}-\lambda_{3} \in Z_{1}$, then, from $\lambda_{5}-\lambda_{4}=\left(\lambda_{5}-\lambda_{3}\right)-\left(\lambda_{4}-\lambda_{3}\right) \in Z_{1}-Z_{1}$, Proposition 2.4 and (3.43), we have $y_{5,4}, z_{5,4} \in \mathbb{Z}$, which contradicts Proposition $2.3(\mathrm{~d})(\mathrm{e})$.

2) If $\lambda_{5}-\lambda_{3}, \lambda_{4}-\lambda_{3} \in Z_{3}$, then, from $\lambda_{5}-\lambda_{4}=\left(\lambda_{5}-\lambda_{3}\right)-\left(\lambda_{4}-\lambda_{3}\right) \in Z_{3}-Z_{3}$, Proposition 2.2(v) and (3.43), we have $y_{5,4}, z_{5,4} \in \mathbb{Z}$, which contradicts Proposition 2.3(d) (e).

Claim 5.2 Any two differences of the above four differences can not belong to the same set $Z_{2}$ or $Z_{3}$. 
Claim 5.2 can be checked directly. For example, if $\lambda_{5}-\lambda_{1}, \lambda_{3}-\lambda_{1} \in Z_{2}, \lambda_{4}-\lambda_{1}, \lambda_{2}-\lambda_{1} \in Z_{3}$, then, we see each set contains elements (or differences) in the following Box 3:

\begin{tabular}{ccc}
\hline$Z_{1}$ & $Z_{2}$ & $Z_{3}$ \\
\hline & $\lambda_{3}-\lambda_{1}, \lambda_{5}-\lambda_{1}$ & $\lambda_{2}-\lambda_{1}, \lambda_{4}-\lambda_{1}$
\end{tabular}

Box 2

Since $\lambda_{5}-\lambda_{3}=\left(\lambda_{5}-\lambda_{1}\right)-\left(\lambda_{3}-\lambda_{1}\right) \in Z_{2}-Z_{2}$ and $\lambda_{4}-\lambda_{2}=\left(\lambda_{4}-\lambda_{1}\right)-\left(\lambda_{2}-\lambda_{1}\right) \in Z_{3}-Z_{3}$, therefore, by applying Proposition 2.2(iv) (v) and Proposition 2.3(d) (e), we have

$$
\lambda_{5}-\lambda_{3} \in Z_{1} \cup Z_{3}, \lambda_{4}-\lambda_{2} \in Z_{1} \cup Z_{2} \text { and } y_{5,3}, z_{4,2} \in \mathbb{Z} \text {. }
$$

We only discuss the following three cases and deduce a contradiction. The other cases can be proved in the same manner.

Case 5.3. $\lambda_{5}-\lambda_{3} \in Z_{3}, \lambda_{4}-\lambda_{2} \in Z_{2}$;

Case 5.4. $\lambda_{5}-\lambda_{3} \in Z_{1}, \lambda_{4}-\lambda_{2} \in Z_{2}$;

Case 5.5. $\lambda_{5}-\lambda_{3} \in Z_{1}, \lambda_{4}-\lambda_{2} \in Z_{1}$.

3) In Case 5.3, From $\lambda_{5}-\lambda_{3}=\left(\lambda_{5}-\lambda_{2}\right)-\left(\lambda_{3}-\lambda_{2}\right) \in Z_{1}-Z_{1}$, Proposition 2.4 and (3.44), we lead to a contradiction.

4) In Case 5.4, we consider the remainder differences

$$
\lambda_{5}-\lambda_{4}, \lambda_{5}-\lambda_{2}, \lambda_{4}-\lambda_{3}, \lambda_{3}-\lambda_{2} \text {. }
$$

Then, any three differences of the above four differences can not belong to the same set $Z_{1}$. In fact, for example, if $\lambda_{5}-\lambda_{2}, \lambda_{4}-\lambda_{3}, \lambda_{3}-\lambda_{2} \in Z_{1}$, then from $\lambda_{4}-\lambda_{2}=\left(\lambda_{4}-\lambda_{3}\right)+\left(\lambda_{3}-\lambda_{2}\right) \in Z_{1}-Z_{1}$, Proposition 2.4 and (3.44), we lead to a contradiction.

5) In Case 5.5, the following consider the remainder differences

$$
\lambda_{5}-\lambda_{4}, \lambda_{5}-\lambda_{2}, \lambda_{4}-\lambda_{3}, \lambda_{3}-\lambda_{2} \text {. }
$$

Then, any two differences of the above four differences can not belong to the same set $Z_{1}$. In fact, for example, if $\lambda_{3}-\lambda_{2}, \lambda_{5}-\lambda_{4} \in Z_{1}$, then since

$$
\begin{aligned}
& \lambda_{5}-\lambda_{2}=\left(\lambda_{5}-\lambda_{3}\right)+\left(\lambda_{3}-\lambda_{2}\right) \in Z_{1}+Z_{1} \\
& \text { and } \lambda_{4}-\lambda_{3}=\left(\lambda_{4}-\lambda_{2}\right)-\left(\lambda_{3}-\lambda_{2}\right) \in Z_{1}-Z_{1},
\end{aligned}
$$

therefore, by applying Proposition 2.4, we have

$$
\lambda_{5}-\lambda_{2}, \lambda_{4}-\lambda_{3} \in Z_{2} \cup Z_{3} \text { and } z_{5,2}-y_{5,2}, z_{4,3}-y_{4,3} \in \mathbb{Z} \text {. }
$$

i) $\lambda_{5}-\lambda_{2}, \lambda_{4}-\lambda_{3}$ can not belong to the same set $Z_{2}$ or $Z_{3}$. The reason is as follows.

For example, if $\lambda_{5}-\lambda_{2}, \lambda_{4}-\lambda_{3} \in Z_{2}$, then from

$$
\begin{aligned}
& \lambda_{4}-\lambda_{1}=\left(\lambda_{4}-\lambda_{3}\right)+\left(\lambda_{3}-\lambda_{1}\right) \in Z_{2}+Z_{2}, \\
& \lambda_{2}-\lambda_{1}=\left(\lambda_{5}-\lambda_{1}\right)-\left(\lambda_{5}-\lambda_{2}\right) \in Z_{2}-Z_{2}
\end{aligned}
$$

and Proposition 2.2(iv), we have

$$
y_{2,1}, y_{4,1} \in \mathbb{Z} \text {. }
$$


Further, from $\lambda_{4}-\lambda_{2}=\left(\lambda_{4}-\lambda_{1}\right)-\left(\lambda_{2}-\lambda_{1}\right) \in Z_{3}-Z_{3}$, Proposition $2.2(\mathrm{v})$ and (3.46), we have $y_{4,2}, z_{4,2} \in \mathbb{Z}$, which contradicts Proposition 2.3(d) (e).

ii) $\lambda_{5}-\lambda_{2}$ can not belong to the set $Z_{2}$ or $Z_{3}, \lambda_{4}-\lambda_{3}$ can not belong to the set $Z_{3}$ or $Z_{2}$. The reason is as follows. For example, if $\lambda_{5}-\lambda_{2} \in Z_{2}, \lambda_{4}-\lambda_{3} \in Z_{3}$, then there let

$$
\left(\lambda_{5}-\lambda_{2}\right)+\left(\lambda_{4}-\lambda_{3}\right)=\left(\xi_{1}, \xi_{2}, \xi_{3}\right)^{\mathrm{T}} \text { and }\left(\lambda_{5}-\lambda_{3}\right)+\left(\lambda_{4}-\lambda_{2}\right)=\left(\eta_{1}, \eta_{2}, \eta_{3}\right)^{\mathrm{T}} \text {. }
$$

By applying Proposition 2.2, Proposition 2.3 and Proposition 2.4, we have

$$
\xi_{2}, \xi_{3} \in \mathbb{Z}, \eta_{2}, \eta_{3} \notin \mathbb{Z}
$$

Namely

$$
\left(\lambda_{5}-\lambda_{2}\right)+\left(\lambda_{4}-\lambda_{3}\right) \neq\left(\lambda_{5}-\lambda_{3}\right)+\left(\lambda_{4}-\lambda_{2}\right),
$$

we immediately deduce a contradiction. This completes the proof of Typical case 5.

According to the above discussion of Typical cases 1 - 5, we proved that any set of $\mu_{M, D}$-orthogonal exponentials contains at most 4 elements, and $\mu_{M, D}$ is a non-spectral measure. One can obtain many such orthogonal systems which contain four elements. For instance, the exponential function system $E_{\Lambda}$ with $\Lambda$ given by

$$
\Lambda=\left\{0, M^{*} s_{1}, M^{*} s_{2}, M^{*} s_{3}\right\},
$$

where

$$
s_{1}=\left(\frac{1}{2}, \frac{1}{2}, 0\right)^{\mathrm{T}}, s_{2}=\left(\frac{1}{2}, 0, \frac{1}{2}\right)^{\mathrm{T}}, s_{3}=\left(0, \frac{1}{2}, \frac{1}{2}\right)^{\mathrm{T}}
$$

is a four-elements orthogonal system in $L^{2}\left(\mu_{M, D}\right)$. This shows that the number 4 is the best. This completes the proof of Theorem 1.1.

By the proof of Theorem 1.1, we have the following corollaries:

Corollary 3.1. For self-affine measure $\mu_{M, D}$ corresponding to the expanding integer matrix

$$
M=\left[\begin{array}{lll}
p_{1} & 0 & 0 \\
p_{2} & p & 0 \\
p_{3} & 0 & p
\end{array}\right] \text { and } D=\left\{\left(\begin{array}{l}
0 \\
0 \\
0
\end{array}\right),\left(\begin{array}{l}
d \\
0 \\
0
\end{array}\right),\left(\begin{array}{l}
0 \\
d \\
0
\end{array}\right),\left(\begin{array}{l}
0 \\
0 \\
d
\end{array}\right)\right\},
$$

if $p, p_{1} \notin 2 \mathbb{Z}$ and $d \neq 0$, then $\mu_{M, D}$ is a non-spectral measure and there exist at most 4 mutually orthogonal exponential functions in $L^{2}\left(\mu_{M, D}\right)$, where the number 4 is the best possible.

Corollary 3.2. For self-affine measure $\mu_{M, D}$ corresponding to the expanding integer matrix

$$
M=\left[\begin{array}{lll}
p & 0 & p_{1} \\
0 & p & p_{2} \\
0 & 0 & p_{3}
\end{array}\right] \text { and } D=\left\{\left(\begin{array}{l}
0 \\
0 \\
0
\end{array}\right),\left(\begin{array}{l}
d \\
0 \\
0
\end{array}\right),\left(\begin{array}{l}
0 \\
d \\
0
\end{array}\right),\left(\begin{array}{l}
0 \\
0 \\
d
\end{array}\right)\right\}
$$

if $p, p_{3} \notin 2 \mathbb{Z}$ and $d \neq 0$, then $\mu_{M, D}$ is a non-spectral measure and there exist at most 4 mutually orthogonal exponential functions in $L^{2}\left(\mu_{M, D}\right)$, where the 
number 4 is the best possible.

\section{A Concluding Remark}

In this section, we would like to point out that the above proof is based on the relations inside the zero set $Z\left(\hat{\mu}_{M, D}\right)$ of the Fourier transform $\mu_{M, D}$. Generally speaking, it is difficult to obtain certain properties on the zero set. If we choose the special four-elements digit set, for instance, the direct-sum-forms digit set

$$
D=D_{1} \oplus D_{2}=\left\{\left(\begin{array}{l}
0 \\
0 \\
0
\end{array}\right),\left(\begin{array}{l}
1 \\
0 \\
0
\end{array}\right)\right\} \oplus\left\{\left(\begin{array}{l}
0 \\
0 \\
0
\end{array}\right),\left(\begin{array}{l}
0 \\
1 \\
0
\end{array}\right)\right\},
$$

it is easy to estimate the number of orthogonal exponentials.

Proposition 4.1. For an expanding integer matrix $M \in M_{3}(\mathbb{Z})$ and the direct-sum-forms digit set $D$ given by

$$
M=\left[\begin{array}{ccc}
p_{1} & 0 & p_{5} \\
0 & p_{2} & p_{4} \\
0 & 0 & p_{3}
\end{array}\right] \text { and } D=\left\{\left(\begin{array}{l}
0 \\
0 \\
0
\end{array}\right),\left(\begin{array}{l}
1 \\
0 \\
0
\end{array}\right),\left(\begin{array}{l}
0 \\
1 \\
0
\end{array}\right),\left(\begin{array}{l}
1 \\
1 \\
0
\end{array}\right)\right\},
$$

if $p_{1}, p_{2} \notin 2 \mathbb{Z}$, then $\mu_{M, D}$ is a non-spectral measure and there exist at most 4 mutually orthogonal exponential functions in $L^{2}\left(\mu_{M, D}\right)$.

Proof. We consider the pair $\left(M, D_{1}\right)$ and $\left(M, D_{2}\right)$, let $\bar{\Theta}_{0}:=\left\{\xi \in \mathbb{R}^{3}: m_{D_{1}}(\xi)=0\right\}$ and let $\tilde{\Theta}_{0}:=\left\{\xi \in \mathbb{R}^{3}: m_{D_{2}}(\xi)=0\right\}$. Then

$$
\begin{aligned}
& \bar{\Theta}_{0}=\left\{\left(\begin{array}{c}
\frac{1}{2}+\bar{k} \\
\bar{\alpha} \\
\bar{\beta}
\end{array}\right): \bar{k} \in \mathbb{Z}, \bar{\alpha}, \bar{\beta} \in \mathbb{R}\right\}, \\
& \left.\tilde{\Theta}_{0}=\left\{\begin{array}{c}
\tilde{\alpha} \\
\frac{1}{2}+\tilde{k} \\
\tilde{\beta}
\end{array}\right): \tilde{k} \in \mathbb{Z}, \tilde{\alpha}, \tilde{\beta} \in \mathbb{R}\right\} .
\end{aligned}
$$

In the case when $p_{1}, p_{2} \notin 2 \mathbb{Z}$, we see that

$$
M^{*} \bar{\Theta}_{0} \in \bar{\Theta}_{0}, M^{*} \tilde{\Theta}_{0} \in \tilde{\Theta}_{0},
$$

which show that

$$
Z\left(\hat{\mu}_{M, D_{1}}\right) \subseteq \bar{\Theta}_{0}, Z\left(\hat{\mu}_{M, D_{2}}\right) \subseteq \tilde{\Theta}_{0} .
$$

If $\lambda_{j}(j=1,2,3) \in \mathbb{R}^{3}$ are such that the exponential functions

$$
\mathrm{e}^{2 \pi i\left\langle\lambda_{1}, x\right\rangle}, \mathrm{e}^{2 \pi i\left\langle\lambda_{2}, x\right\rangle}, \mathrm{e}^{2 \pi i\left\langle\lambda_{3}, x\right\rangle}
$$

are mutually orthogonal in $L^{2}\left(\mu_{M, D_{1}}\right)$, then the differences $\lambda_{j}-\lambda_{i}(1 \leq i \neq j \leq 3)$ are in the zero set $Z\left(\hat{\mu}_{M, D_{1}}\right)$. That is, there are $\bar{k}_{1}, \bar{k}_{2}, \bar{k}_{3} \in \mathbb{Z}, \bar{\alpha}_{1}, \bar{\alpha}_{2}, \bar{\alpha}_{3} \in \mathbb{R}$ and $\bar{\beta}_{1}, \bar{\beta}_{2}, \bar{\beta}_{3} \in \mathbb{R}$ such that 


$$
\lambda_{3}-\lambda_{1}=\left(\begin{array}{c}
\frac{1}{2}+\bar{k}_{1} \\
\bar{\alpha}_{1} \\
\bar{\beta}_{1}
\end{array}\right), \lambda_{2}-\lambda_{1}=\left(\begin{array}{c}
\frac{1}{2}+\bar{k}_{2} \\
\bar{\alpha}_{2} \\
\bar{\beta}_{2}
\end{array}\right), \lambda_{3}-\lambda_{2}=\left(\begin{array}{c}
\frac{1}{2}+\bar{k}_{3} \\
\bar{\alpha}_{3} \\
\bar{\beta}_{3}
\end{array}\right) .
$$

This shows that

$$
\left(\lambda_{3}-\lambda_{1}\right)-\left(\lambda_{2}-\lambda_{1}\right) \neq \lambda_{3}-\lambda_{2}
$$

a contradiction. Hence, there are at most 2 mutually orthogonal exponential functions in $L^{2}\left(\mu_{M, D_{1}}\right)$.

Similarly, there are at most 2 mutually orthogonal exponential functions in $L^{2}\left(\mu_{M, D_{2}}\right)$.

From

$$
\hat{\mu}_{M, D}(\xi)=\hat{\mu}_{M, D_{1}}(\xi) \hat{\mu}_{M, D_{2}}(\xi)
$$

we have

$$
Z\left(\hat{\mu}_{M, D}\right)=Z\left(\hat{\mu}_{M, D_{1}}\right) \cup Z\left(\hat{\mu}_{M, D_{2}}\right) .
$$

Hence $\mu_{M, D}$ is a non-spectral measure, and there are at most 4 mutually orthogonal exponential functions in $L^{2}\left(\mu_{M, D}\right)$.

\section{Conflicts of Interest}

The author declares no conflicts of interest regarding the publication of this paper.

\section{References}

[1] Hutchinson, J.E. (1981) Fractals and Self-Similarity. Indiana University Mathematics Journal, 30, 713-747. https://doi.org/10.1512/iumj.1981.30.30055

[2] Fuglede, B. (1974) Commuting Self-Adjoint Partial Differential Operators and a Group Theoretic Problem. Journal of Functional Analysis, 16, 101-121. https://doi.org/10.1016/0022-1236(74)90072-X

[3] Kolountzakis, M.N. (2000) Non-Symmetric Convex Domains Have No Basis of Exponentials. Illinois Journal of Mathematics, 44, 542-550. https://doi.org/10.1215/ijm/1256060414

[4] Fuglede, B. (2001) Orthogonal Exponentials on the Ball. Expositiones Mathematicae, 19, 267-272. https://doi.org/10.1016/S0723-0869(01)80005-0

[5] Jorgensen, P.E.T. and Pedersen, S. (1998) Dense Analytic Subspaces in Fractal $L^{2}$ Spaces. Journal d Analyse Mathematique, 75, 185-228. https://doi.org/10.1007/BF02788699

[6] Iosevich, A., Katz, N. and Tao, T. (2001) Convex Bodies with a Point of Curvature Do Not Have Fourier Basis. American Journal of Mathematics, 123, 115-120. https://doi.org/10.1353/ajm.2001.0003

[7] Wang, Z.Y. and Liu, J.C. (2019) Non-Spectrality of Self-Affine Measures. Journal of Functional Analysis, 277, 3723-3736. https://doi.org/10.1016/j.jfa.2019.05.015

[8] Liu, J.C., Dong, X.H. and Li, J.L. (2017) Non-Spectral Problem for the Planar Self-Affine Measures. Journal of Functional Analysis, 273, 705-720. 
https://doi.org/10.1016/j.jfa.2017.04.003

[9] Dudkay, D.E. and Jorgensen, P.E.T. (2007) Analysis of Orthogonality and of Orbits in Affine Iterated Function Systems. Mathematische Zeitschrift, 256, 801-823. https://doi.org/10.1007/s00209-007-0104-9

[10] Łaba, I. and Wang, Y. (2002) On Spectral Cantor Measures. Journal of Functional Analysis, 193, 409-420. https://doi.org/10.1006/jfan.2001.3941

[11] Strichartz, R. (2000) Mock Fourier Series and Transforms Associated with Certain Cantor Measures. Journal d Analyse Mathematique, 81, 209-238. https://doi.org/10.1007/BF02788990

[12] Strichartz, R. (2006) Convergence of Mock Fourier Series. Journal d Analyse Mathematique, 99, 333-353. https://doi.org/10.1007/BF02789451

[13] Wang, Q. and Li, J.L. (2019) There Are Eight-Element Orthogonal Exponentials on the Spatial Sierpinski Gasket. Mathematische Nachrichten, 292, 211-226. https://doi.org/10.1002/mana.201700471

[14] Li, J.L. (2008) Non-Spectral Problem for a Class of Planar Self-Affine Measures. Journal of Functional Analysis, 255, 3125-3148. https://doi.org/10.1016/j.jfa.2008.04.001

[15] Li, J.L. (2009) Non-Spectrality of Planar Self-Affine Measures with Three-Elements Digit Set. Journal of Functional Analysis, 257, 537-552.

https://doi.org/10.1016/j.jfa.2008.12.012

[16] Wang, Q. and Li, J.L. (2020) The Maximal Cardinality of $\mu_{M, D}$-Orthogonal Exponentials on the Spatial Sierpinski Gasket. Monatshefte für Mathematik, 191, 203-224. https://doi.org/10.1007/s00605-019-01348-9

[17] Yuan, Y.B. (2009) Orthogonal Exponentials on the Generalized Three Dimension Sierpinski Gasket. Journal of Mathematical Analysis and Applications, 349, 395-402. https://doi.org/10.1016/j.jmaa.2008.08.047

[18] Hu, T.Y. and Lau, K.S. (2008) Spectral Property of the Bernoulli Convolutions. Advances in Mathematics, 219, 554-567. https://doi.org/10.1016/j.aim.2008.05.004 